\title{
Comment on fungal tape lift reporting frameworks
}

\section{Introduction}

The microbiology of the built environment and its relationship to occupant health indoors is an increasingly active area of scholarship. ${ }^{1}$ For example, adverse human-microbe interactions are often claimed as the cause of sick-building syndrome-type complaints; while it is argued that different indoor habitats including inanimate surfaces confer selection pressure on common environmental fungi leading towards increasing virulence. ${ }^{2}$ In turn, measuring actual or suspect indoor fungal contamination is increasingly common especially on indoor damp surfaces ${ }^{3}$ or following water damage, ${ }^{4}$ and is an ongoing area of occupational health and safety and the focus of building disputes and litigation. ${ }^{5}$ While there are established metrics for assessing the microbiological component of air using viable colony counts or viable and non-viable fungal spore counts in units per cubic meter of air; ${ }^{6,7}$ there is some difficulty with how best to measure and report surface fungal contamination using tape lifts where there is no consensus unit used for reporting. The method of using sticky tape to transfer fungal colonies from one surface onto microscope slides was first reported for dermatophyte fungi in the 1970's and later for other fungi. ${ }^{89}$ This was shown to be an excellent technique that preserved for example the conidium and conidiophore morphology. Many researchers still use sticky tape sampling to evaluate biological contaminants like indoor fungi. ${ }^{10-12}$ while the method has proven valuable in criminal and civil forensics where mould growth or absence has been used as evidence linking people and objects with places ${ }^{13}$ or for sampling of other contaminants like chemical threat agents. ${ }^{14}$ The commercial development of readily available and inexpensive tape lifts, ${ }^{15,16}$ offer a consistent sample area on a flexible plastic slide for the determination of mould, other microbial, settled bioaerosols, and inorganic dust contamination. However, the problem of 'describing what is seen' remains. This has a lot to do with the diversity of locations that samples may come from and from the type of information that is sought. Eight methods have been identified. Each has more or less merit depending on the amount and quality of fungal material versus background debris. The first aim of this comment is therefore to review the literature and offer an opinion about a standardized protocol for fungal surface testing using tape lifts. The second aim is to offer the analyst a flexible framework when choosing an appropriate qualitative or semi-quantitative reporting index that is matched both to the quality and objectives of the sampling. Usually the goal is to identify a healthy or unhealthy building microbiome and to guide the scope or validate any remediation effort that has or should or must occur (IICRC S500/S520/R520). ${ }^{17,18}$

\section{Method I}

Two current Standards focus on sampling surfaces for fungi using tape lifts. The first ${ }^{19}$ (D7910-14) is a Standard Practice that states that tape lifts may be used for both "qualitative or quantitative analysis by direct microscopy" of "material present at one specific location on a surface for fungal content". The significance of use is for "qualitative analysis or to quantify fungal material per sample or per unit area". These statements are entirely sensible since the recovery efficiency is a source of uncertainty and the Standard does not comment on sampling objectives or how the method is used to address building

\author{
Volume 7 Issue 6 - 2019
}

\author{
Cameron L Jones', \\ 'Biological Health Services, Australia \\ ${ }^{2}$ National Institute of Integrative Medicine, Australia
}

Correspondence: Dr. Cameron L. Jones, Biological Health Services, Level I, 459 Toorak Rd,Toorak,Victoria, 3142, Australia, Tel+61414998900, Email inf@biologicalhealthservices.com.au

Received: November 26, 2019 | Published: December 10, 2019

occupant exposure or occupant health risk. The second ${ }^{20}$ (D7658-17), is a Standard Test Method and the significance of use is to ensure consistency between laboratories and analysts, where "Fungal structures are identified and semi-quantified regardless of whether they would or would not grow in culture". As well, the intention of the Standard "is to standardize the analysis of the detection of removable fungal structures lifted from a surface with tape". The analyst will then "determine and record each fungal type as encountered" into 12 minimum categories including into Genus and spore type morphologies as seen. The problem is that depending on how the sample was taken, where it was taken from, and how much vegetative material is present, will all impact on the type and accuracy of information that is possible to meaningfully obtain from each slide. The Standard (D7658-17) is unclear since the only quantitative scale offered is a semi-quantitative fungal loading category scale from $0-5$ where: Category $0=$ no fungal material present; Category 1 = fungal material covers $<5 \%$ of a representative field of view; Category $2=5-25 \%$; Category $3=25$ $75 \%$; Category $4=75-90 \%$ and Category $5=>90 \%$. The reference in the Standard D7658-17 is for particle loading of debris but seems to be used interchangeably. Notably, this same approach was adopted for spore counting in the superseded ${ }^{21}$ and current Standard ${ }^{22}$ (D7391-09 and D7391-17 $\mathrm{e}^{1}$ ) where the percentage debris scale defined above was used for recording background debris, not to be mistaken for the spore numbers. To some extent, the objectivity goal of the two Standards for tape lifts falls short since in practice there are several other approaches for microscope-image classification.

\section{Method 2}

The second method for describing contamination according to the Australian Mould Guideline ${ }^{6}$ proposes a Hygiene Rating using 5 categories: Low $=<50$ spores $/ \mathrm{cm}^{2}$; Normal $=50-500$ spores $/ \mathrm{cm}^{2}$; Elevated $=500-1000$ spores $/ \mathrm{cm}^{2}+$ prevailing species; Contaminated $=>1000$ spores $/ \mathrm{cm}^{2}+$ dominant species + propagules; Extreme Contamination $=>5000$ spores $/ \mathrm{cm}^{2}+$ dominant species + propagules + confluent spores. Implementations of this scale have sometimes described the last two categories as 'High' and 'Extremely High' respectively. 


\section{Method 3}

A third method for evaluating contamination was proposed by Krause \& Hammad $^{23}$ who used 4 categories: Category I = described as a "clean surface" $=<10$ fungal structures $/ \mathrm{cm}^{2}$; Category II= described as "light deposition of fungal structures including hyphal fragments and spores" $=100-1000$ fungal structures $/ \mathrm{cm}^{2}$; Category III $=$ described as "accumulation of fungal structures" $=100-1000$ fungal structures $/ \mathrm{cm}^{2}$; Category IV = described as "heavy accumulation of fungal structures and possible amplification" $=>1000$ fungal structures $/ \mathrm{cm}^{2}$. The presence of conidiophores and hyphae are used as indicators of past or present fungal growth.

\section{Method 4}

Another approach used by the American Industrial Hygiene Association (see: Clarke, GA. 2001) ${ }^{24}$ used 3 categories: Normal background $=$ no significant fungal material or no significant fungal biomass showing no more than 1-5\%; Possible contamination source $=5-25 \%$; and Probable contamination source $=25-100 \%$. This has also been adopted by the NYCOSH. ${ }^{25}$

\section{Method 5}

Another percentage-based method ${ }^{26}$ to determine if contents are contaminated by mould and that has been suggested for use as part of post-remediation evaluation sampling or third-party postremediation verification is the following. Surface samples by tape lift can be "analyzed so that the quantity of fungal spores is presented as a percentage of the sample area", "rather than a raw count" where: Normal fungal ecology $=\leq 1 \%$; Indoor environment contaminated with settled spores that were dispersed directly or indirectly (Condition 2 ) = between 1 and 3\%; Indoor environment contaminated with the presence of actual mold growth and associated spores (Condition 3) $=\geq 3 \%$ The presence of target spore types (Chaetomium, Fusarium, Memnoniella, Stachybotrys, and Trichoderma) is an automatic indication of fungal contamination, regardless of the percentage of spores.

\section{Method 6}

Similar interpretations for evaluating toxigenic fungi and mycotoxins in outdoor, recreational environments have been used, ${ }^{27}$ where "the amount of fungal spores was rated based on the coverage of spores on the tape samples observed": Trace $=<5 \%$; Light $=5-25 \%$; Moderate $=26-75 \%$; Heavy $=76-90 \%$ and Very Heavy $=>90 \%$. Another variation uses: Low $=1-25 \%$, Medium $=26-50 \%$, High $=$ $51-75 \%$, Very High $=76-100 \%$ that broadly follows the D7391-09 Standard for estimation of non-microbial particle debris rating. ${ }^{21}$

\section{Method 7}

Other methods ${ }^{28}$ describe the benefits of tape lifts where they are used to test "discolorations resulting from moisture damage [that] may imply mould growth" and are "used to confirm that fungal growth has been removed following remediation". The overall objective is to determine whether mould is present or not? The authors stress that care must be taken not to inappropriately extrapolate from the tape lift area to larger areas that may have different moisture or fungal growth conditions. The method is understood to be "qualitatively specific" and "semi-quantitative at best". Notably, results from interpretation should be "approached with caution" and "laboratory reports should not state results in terms of number of spores per unit area, because the measure is meaningless." The value of the method lies in being able to differentiate between normal accumulation of mould, unusual accumulation of spores linked to adjacent fungal growth or confirmation of fungal growth on a surface. Two sets of categories are proposed: No spores detected; No abundance of unusual types (those types often associated with growth on building materials); minimal abundance of unusual types; moderate abundance of unusual types, and; high abundance of unusual types. The second category scale is: $0=$ no growth $1+=$ minimal fungal growth; $2+=$ low to moderate fungal growth; $3+=$ moderate fungal growth; $4+=$ heavy fungal growth.

\section{Method 8}

One final descriptive scale for tape lifts was advanced by Horner et al. ${ }^{29}$ where the presence or absence of spores, hyphae and fruiting structures were recorded against common Taxa from: Alternaria, Chaetomium, Eurotium, Cladosporium, Penicillium/Aspergillus, Stachybotrys. Spore amounts were noted as: $\mathrm{S}=$ scattered single, $\mathrm{F}$ = few, $\mathrm{A}=$ Abundant or $\mathrm{M}=$ massive. Amounts of hyphae/fruiting structures were noted as: $\mathrm{S}=$ scattered single, $\mathrm{F}=$ few, or $\mathrm{A}=$ abundant.

\section{Conclusion}

From the above 8 methods, we see that describing tape lift observations is sometimes objective and sometimes subjective. There is uncertainty in tape lift data because there is variance in: (i) the area or region of interest being sampled, (ii) the number of samples, (iii) the hypothesis being tested (i.e. is the expected outcome clean or unclean) ${ }^{30}$ (iv) the experience of the sampler, (v) how much pressure was used to take the sample, (vi) how much background debris was present, (vii) the relationship between the tape lift data and other metrics of microbial exposure (surface and airborne) and potentially (viii) cost factors. Sampling too few surfaces or selectively choosing or avoiding locations could skew or bias the data set. Similarly, only relying on tape lifts for surface contamination measurement without performing companion testing like RODAC contact plates or swab testing to viable culture or surrogate surface cleanliness metrics like ATP bioluminescence could allow for a lack of convergence when assessing all the surface data and lead to interpretation and recommendation errors. With this in mind, I propose that tape lift fungal assessments should use a combination of several (at least 3) of the above methods that are well-matched to the observed structures seen under the microscope. Including representative micrographs in reports could also be helpful. This will improve reliability and validity of surface sampling for fungi. There is also considerable risk in over-objectifying subjective microscope-data, especially when image-analysis is not used. This can occur when lab reports are produced showing extensive fungal Speciation categorized into fine-grained spore or fungal structures. Apart from going against the advice from the AIHA, ${ }^{28}$ the relationship between the human observer and how biased judgment and misrepresentation can occur should not be underestimated. Excepting for tape transfers made from surfaces showing abundant visual mould growth (like biofilms or surfaces showing visual bulk), there is little chance that strict numerate taxonomic rankings can be reported. It is undisputed that tape lifts are useful in industrial hygiene water damage investigations or for environmental screening of contaminated surfaces and objects ${ }^{31,32}$ or to assess for potential fungal particle transmission. ${ }^{33}$ However, the value of this method of measurement is a continuum between the objective and subjective and depends on the overall image quality of the fungi that can be removed by the adhesive from any chosen surface. 


\section{Funding details}

None.

\section{Acknowledgements}

None.

\section{Conflicts of interest}

The author declares no conflict of interest.

\section{References}

1. Adams RI, Bhangar S, Dannemiller KC, et al. Ten questions concerning the microbiomes of buildings. Build Environ. 2016;109: 224-234.

2. Gostincar C, Grube M, Gunde-Cimerman N. Evolution of fungal pathogens in domestic environments? Fungal Biol-UK. 2011; 1008-1018.

3. Habibi A, Safaierfarahani B. Indoor damp surfaces harbor molds with clinical significance. Curr Med Mycol. 2018;4(3): 1-9.

4. WHO. WHO guidelines for indoor air quality: dampness and mould. 2009.

5. Jones CL. Mould in building disputes. J Bacteriol Mycol Open Access. 2018. 6(4): 264-272.

6. Kemp P, Neumeister-Kemp H. Australian Mould Guideline; the Go-to Guide for Everything Mould. 2nd edn. Sydney. Messenger Publishing; 2010

7. Jones CL. Crowd sourced taxonomic identification guide for categorization and quantification of fungal spores by optical microscopy. $J \mathrm{Mycol}$ Mycological Sci. 2019;2(1):000106.

8. Flegal TW. Semipermanent microscope slides of microfungi using a sticky tape technique. Can J Microbiol. 1980;26:551-553.

9. St-Germain G, Summerbell R. Identifying Filamentous Fungi, A Clinica Laboratory Handbook. California: Star Publishing Company; 1996.

10. Portnoy JM, Barnes CS, Kennedy K. Sampling for indoor fungi. J Allergy Clin Immunol. 2004;113(2):189-198.

11. Thomas G, Burton NC, Mueller C et al. Comparison of work-related symptoms and visual contrast sensitivity between employees at a severely water-damaged school and a school without significant water damage. $\mathrm{Am}$ J Ind Med. 2012;55(9):844-854.

12. Hung LL, Miller JD, Dillon HK, et al. Field Guide for the Determination of Biological Contaminants in Environmental Samples. 2nd edn. Fairfax (VA): The American Industrial Hygiene Association; 2005.

13. Hawksworth DL, Wiltshire PEJ. Forensic mycology: current perspectives. Research and Reports in Forensic Medical Science. 2015;5:75-83.

14. Brady K, Stilley B, Olds M. Tape lift sampling of chemical threat agents. J Forensic Sci. 2017;62(4):1015-1021.

15. Zefon Bio-Tape.
16. Stick-to-it Lift Tape. SKC Inc.

17. Institute of Inspection Cleaning and Restoration Certification. ANSI/ IICRC S520 Standard and IICRC R520 Reference Guide for Professional Mold Remediation. 2015.

18. Institute of Inspection Cleaning and Restoration Certification. ANSI/ IICRC S500 Standard and Reference Guide for Professional Water Damage Restoration. 2015.

19. ASTM D7910-14. Standard practice for collection of fungal material from surfaces by tape lift.

20. ASTM D7658-17. Standard test method for direct microscopy of fungal structures from tape.

21. ASTM D7391 - 09. Standard test method for categorization and quantification of airborne fungal structures in an inertial impaction sample by optical microscopy.

22. ASTM D7391 - 17e1. Standard test method for categorization and quantification of airborne fungal structures in an inertial impaction sample by optical microscopy.

23. Krause JD, Hammad YY. Measuring the efficacy of mold remediation on contaminated ductwork. Proceedings: Indoor Air. 2002;360-365.

24. Clark GA. Assessment \& Sampling Approaches for Indoor Microbial Assessments. The Synergist (AIHA). 2001.

25. NYCOSH. Methods for Evaluation of Indoor Mold Growth. New York Committee for Occupational Safety and Health.

26. Pinto MA. Mold testing: The old, the new, the useful. Cleaning \& Restoration. 2012;49(30):38-43.

27. Sudakin D, Fallah P. Toxigenic fungi and mycotoxins in outdoor, recreational environments. Clin Toxicol. 2008;46:738-744.

28. Prezant B, Weekes DM, Miller JD. Recognition, Evaluation, and Control of Indoor Mold. Fairfax (VA): The American Industrial Hygiene Association; 2008.

29. Horner WE, Barnes C, Codina R, et al. Guide for interpreting reports from inspections/investigations of indoor mold. J Allergy Clin Immunol. 2008;121(3):592-597

30. Bloomfield SF, Stanwell-Smith R, Crevel RWR, et al. Too clean, or not to clean: the Hygiene Hypothesis and home hygiene. Clin Exp Allergy. 2006;36(4):402-425.

31. Wilson SC, Palmatier RN, Andriychuk LA, et al. Mold contamination and air handling units. J Occup Environ Hyg. 2007;4(7): 483-491.

32. Shirakawa MA, Gaylarde CC, Gaylarde PM, et al. Fungal colonization and succession on newly painted buildings and the effect of biocide. FEMS Microbiology Ecology. 2002;39:165-173.

33. Meheust D, Le Cann P, Reboux G, et al. Indoor fungal contamination: health risks and measurement methods in hospitals, homes and workplaces. Crit Rev Microbiol. 2013; 40(3):1-13. 